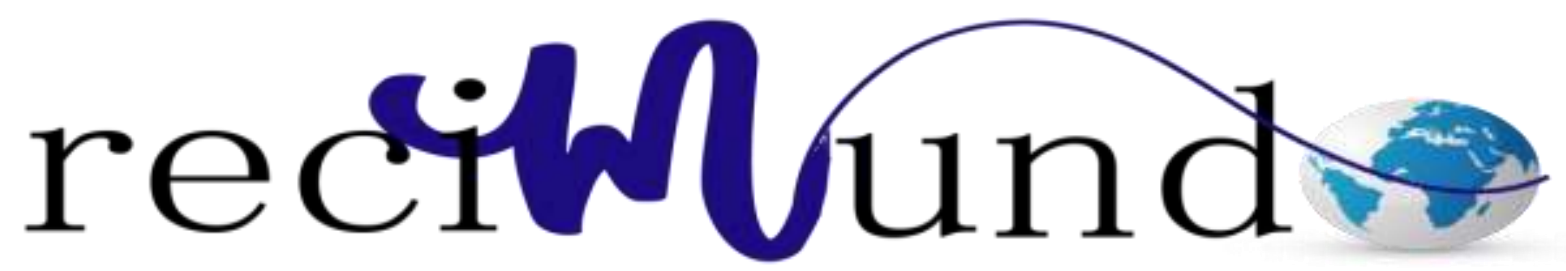

Revista Cientifica Mundo de la Investigación y el Conocimiento

María José Cajas Romero a; Pedro Andrés Duque Torres ${ }^{\text {b; }}$ Margarita Alexandra Guagalango Cabrera ${ }^{\mathrm{c}}$; Karen Paola Barragan Arias ${ }^{\mathrm{d}}$

Taponamiento nasal en epistaxis posterior

Nasal tamponade in posterior epistaxis

Revista Científica Mundo de la Investigación y el Conocimiento. Vol. 3 núm.3, septiembre, ISSN: 2588-073X, 2019, pp. 461-476

DOI: $10.26820 /$ recimundo/3.(3).septiembre.2019.461-476

URL: http://recimundo.com/index.php/es/article/view/531

Código UNESCO: 3205 Medicina Interna

Tipo de Investigación: Artículo de Revisión

Editorial Saberes del Conocimiento

Recibido: 15/05/2019

Aceptado: 23/06/2019

Publicado: 30/09/2019

Correspondencia: majocajas93@gmail.com

a. Médico; Investigador Independiente; Guayaquil, Ecuador; majocajas93@ gmail.com

b. Médico; Investigador Independiente; Guayaquil, Ecuador; padt.2005@gmail.com

c. Médico; Investigador Independiente; Guayaquil, Ecuador;alexamagui@ hotmail.com

d. Médico; Investigador Independiente; Guayaquil, Ecuador; karenpaola.kb@gmail.com 


\section{Taponamiento nasal en epistaxis posterior}

Vol. 3, núm. 3., (2019)

María José Cajas Romero; Pedro Andrés Duque Torres; Margarita Alexandra Guagalango Cabrera; Karen Paola Barragan Arias

\section{RESUMEN}

La epistaxis se trata de un signo clínico y no de una enfermedad, el cual es un motivo de consulta extremadamente frecuente en los servicios de urgencias y atención primaria, pudiendo presentarse tanto en niños como en adultos, con una incidencia de distribución bimodal, mayormente en niños y jóvenes, y en adultos mayores (45-65 años). Para solucionar este cuadro clínico se recurre el uso del taponamiento nasal el cual es una maniobra de obligado aprendizaje y que acostumbra a ser realizada por la facultad de especialistas. Los pacientes con taponamiento posterior necesitan ser mantenidos en observación, ya que pueden presentar dificultad respiratoria. Los pacientes con que ha sido tratado con este tipo de procedimiento deben seguir una serie de recomendaciones que los ayudara a mejorar su cuadro clínico. Cabe mencionar que en lo que va del siglo XXI se ha experimentado una significativa expansión en alternativas para el manejo de las epistaxis. Si bien es cierto, las tradicionales técnicas de taponamiento nasal aún se realizan con éxito, y con evidente satisfacción de los pacientes.

Palabras Claves: Epistaxis; Taponamiento; Endoscópico; Nasal; Posterior. 


\title{
Taponamiento nasal en epistaxis posterior
}

Vol. 3, núm. 3., (2019)

María José Cajas Romero; Pedro Andrés Duque Torres; Margarita Alexandra Guagalango Cabrera; Karen Paola Barragan Arias

\begin{abstract}
Epistaxis is a clinical sign and not a disease, which is an extremely frequent reason for consultation in the emergency and primary care services, and can occur both in children and adults, with an incidence of bimodal distribution, mostly in children and young people, and in older adults (45-65 years). To solve this clinical picture, the use of nasal tamponade is used, which is a compulsory learning maneuver and is usually performed by the faculty of specialists. Patients with subsequent tamponade need to be kept under observation, since they may present respiratory distress. Patients who have been treated with this type of procedure should follow a series of recommendations that will help them improve their clinical picture. It is worth mentioning that so far in the 21 st century there has been a significant expansion in alternatives for the management of epistaxis. While it is true, traditional nasal plugging techniques are still performed successfully, and with obvious patient satisfaction.
\end{abstract}

Key Words: Epistaxis; Plugging; Endoscopic; Nasal; Later. 


\section{Taponamiento nasal en epistaxis posterior}

Vol. 3, núm. 3., (2019)

María José Cajas Romero; Pedro Andrés Duque Torres; Margarita Alexandra Guagalango Cabrera; Karen Paola Barragan Arias

\section{Introducción.}

La epistaxis se trata de un signo clínico y no de una enfermedad, el cual es un motivo de consulta extremadamente frecuente en los servicios de urgencias y atención primaria, pudiendo presentarse tanto en niños como en adultos, con una incidencia de distribución bimodal, mayormente en niños y jóvenes, y en adultos mayores (45-65 años). Por su frecuencia y su potencial gravedad, se considera importante la necesidad de conocer la irrigación nasal y los diferentes métodos para cohibir todo tipo de hemorragia, ya que puede llevar a alteraciones hemodinámicas, que requieran incluso de hospitalización. Además, recordando que es un signo, es importante buscar la causa o enfermedad que la produce, para así asegurar un tratamiento efectivo. (Calderòn, Mairena, \& Mata, 2014)

La epistaxis, definida como un episodio de sangrado activo por la nariz, corresponde al motivo de consulta en urgencia más frecuente en otorrinolaringología. Se estima que aproximadamente el $60 \%$ de la población sufre algún cuadro de epistaxis en su vida, aunque sólo el 6\% de ellos requerirá atención médica para tratamiento y control hemostático, siendo hospitalizados para tal efecto sólo 1,6 por cada 100.000 pacientes. Su incidencia varía con la edad, presentando una distribución bimodal con un peak en niños y jóvenes, y otro en adultos mayores (45-65 años).

La causa de las epistaxis es multifactorial, y resulta de la interacción de una serie de factores ambientales, locales o sistémicos que afectan la mucosa nasal y los vasos sanguíneos. Frecuentemente, como resultado de causas traumáticas o mecánicas, la epistaxis puede progresar a ser incontrolable, o sea aquella hemorragia significativa que requiere asistencia médica para su 


\section{Taponamiento nasal en epistaxis posterior}

Vol. 3, núm. 3., (2019)

María José Cajas Romero; Pedro Andrés Duque Torres; Margarita Alexandra Guagalango Cabrera; Karen Paola Barragan Arias

control. En casos de epistaxis persistente, el rápido y adecuado manejo de la condición inicial del paciente es importante para minimizar la morbilidad y la mortalidad. (Alzérreca, León, Boettiger, \& Naser, 2012)

\section{Materiales y Métodos.}

Las herramientas y materiales utilizados que se conciben para el desarrollo de este trabajo investigativo son los siguientes: computadores personales con conexión a internet y un cúmulo de contenidos científico-académicos diversos, con los que se busca aportar información de calidad y lo más actualizado posible respecto al Taponamiento nasal en epistaxis posterior. Esta investigación está enfocada en la búsqueda y revisión sistemática de literatura científicoacadémica disponible, que puede encontrarse en estas determinadas bases de datos, entre las que figuran: MedlinePlus, PubMed, Biblioteca Virtual de la Salud (BVS), SciELO, Dialnet y ELSEVIER, Cochrane, entre otras; representando todo ello parte esencial del proceso investigativo y de comprensión que facilita la realización de una síntesis concreta de la mejor evidencia disponible, y a su vez representando con esto los resultados esperados.

Principalmente se realiza una búsqueda aleatoria y consecutiva en las mencionadas bases de datos, usando las expresiones "taponamiento nasal", "endoscopía", y "posterior", lo que aproximadamente resultó en miles de registros bibliográficos. Luego éstos se filtran en base a criterios de: idioma español, relevancia, correlación temática y fecha de publicación en lo que va de siglo XXI, sin descartar por qué tipo de material bibliográfico sea; es decir, se escogen títulos de artículos científicos, ensayos, revisiones sistemáticas, tesis de grado, posgrado y doctorado, noticias científicas, entre otros documentos e información de interés científico y académico. 


\section{Taponamiento nasal en epistaxis posterior}

Vol. 3, núm. 3., (2019)

María José Cajas Romero; Pedro Andrés Duque Torres; Margarita Alexandra Guagalango Cabrera; Karen Paola Barragan Arias

Seguidamente, se efectúa la selección y clasificación de la literatura científica académica disponible, aplicando, en términos generales, los criterios antes mencionados, siendo a partir de allí que el equipo investigador le da la correspondiente lectura crítica y análisis de toda esa evidencia científica, lo que resultó consecutiva y consensuadamente en el fundamento de las ideas y planteamientos aquí plasmados.

\section{Resultados.}

Lleva el nombre de epistaxis a todas aquellas pequeñas hemorragias que se producen al dañarse el revestimiento interior o mucosa de la nariz, habitualmente en la zona anterior del tabique nasal. El nombre viene del sustantivo griego é $\pi i ́ \sigma \tau \alpha \xi 1 \varsigma=$ epistaxis que significa fluir gota a gota. (Sociedad epañola de medicina interna, 2019)

El taponamiento nasal es una maniobra de obligado aprendizaje y acostumbrada realización por la facultad de especialistas. En el paciente con epistaxis no siempre se localiza un foco sangrante accesible con rinoscopia anterior y la coexistencia de diversos factores pueden convertir una hemorragia aparentemente banal en una situación amenazante grave. En los Servicios de Urgencias, la epistaxis posterior o incoercible obliga a una acción rápida con gasas y/o materiales expandibles o hinchables. Esta acción a menudo resulta eficaz y obvia que la realización de procedimientos más agresivos.

Los taponamientos para epistaxis posteriores responden a una necesidad no muy frecuente en la asistencia especializada. De hecho, la apreciación de que un tapón de gasa posterior emplea claramente más tiempo en su colocación que un sistema bicameral de inflado pues resulto el principal condicionante para que este último fuera escogido ante epistaxis 


\section{Taponamiento nasal en epistaxis posterior}

Vol. 3, núm. 3., (2019)

María José Cajas Romero; Pedro Andrés Duque Torres; Margarita Alexandra Guagalango Cabrera; Karen Paola Barragan Arias

masivas o inicialmente incontrolables. Por ello los pacientes con desordenes hemostáticos o crisis hipertensivas son preferiblemente taponados con sistemas de inflado, si bien solo se notó una diferencia significativa a nivel estadístico en pacientes con trastornos de la coagulación. Sin embargo, la maniobra del taponamiento posterior resulta incómoda por lo que los relieves internos de las fosas nasales dificultan la introducción de cualquier dispositivo. Aunque el taponamiento con gasas impregnadas con cavum, resulta ser más incómodo en su colocación y mantenimiento, este género menor incidencia de resangrado mientras estuvo colocado, así como tras su retirada en los meses siguientes, existiendo menos casos que precisaran otras alternativas hemostáticas, menor necesidad de trans-fusión de hemoderivados y un número significativamente menor de complicaciones a largo plazo. (García et al., 2010)

La cavidad nasal está dividida en 2 áreas: la cavidad nasal anterior y la cavidad nasal superior.

La irrigación de la región posterior está compuesta principalmente por ramas de la esfenopalatina y la arteria palatina descendente, ambas ramas de la arteria maxilar (carótida externa). También contribuye la arteria etmoidal posterior (carótida interna). Hemorragias de esta zona son más difíciles de localizar y visualizar, dificultando su control.

Según el origen anatómico de la hemorragia, se divide en epistaxis anteriores y posteriores.

Las epistaxis anteriores son las más frecuentes (90-95\%). La mayoría delos sangrados del 


\section{Taponamiento nasal en epistaxis posterior}

Vol. 3, núm. 3., (2019)

María José Cajas Romero; Pedro Andrés Duque Torres; Margarita Alexandra Guagalango Cabrera; Karen Paola Barragan Arias

área de Little son auto limitados. Aquellos sangrados que requieren tratamiento, son fácilmente controlados con medidas locales y conservadoras (cauterización y/o taponamiento nasal anterior mínimo).

Los sangrados posteriores ocurren en un 5 a $10 \%$ de los casos. Se originan más frecuentemente de la arteria esfenopalatina, siendo más común en ancianos y requiriendo a menudo taponamiento nasal anterior y posterior.

Hablamos de epistaxis no complicada, aquélla que logra ser manejada en forma satisfactoria mediante medidas conservadoras como taponamiento nasal (anterior y/o posterior), cauterización química o eléctrica y uso de vasoconstrictores. (Naser \& Aedo, 2007)

\section{Técnicas para el Control de Hemorragia Posterior}

Los pacientes con taponamiento posterior necesitan ser mantenidos en observación, ya que pueden presentar dificultad respiratoria.

Preparar el taponamiento posterior.

- Enrollar la gasa vaselinada de 7,5 x $90 \mathrm{~cm}$, muy apretada, formando un cilindro de 7,5 cm de longitud.

- Atar con cinta umbilical el centro del rollo, dejando los extremos de la cinta largos.

- Atar la segunda cinta umbilical en el mismo punto, dejando también los extremos largos.

- Impregnar el tapón con pomada poli antibiótica.

Inserción del taponamiento 


\section{Taponamiento nasal en epistaxis posterior}

Vol. 3, núm. 3., (2019)

María José Cajas Romero; Pedro Andrés Duque Torres; Margarita Alexandra Guagalango Cabrera; Karen Paola Barragan Arias

- Pasar uno de los catéteres flexibles por la fosa nasal sana, tirando de la punta desde la faringe y sacándola por la boca.

- Pinzar los dos extremos juntos.

- Pasar el segundo catéter del mismo modo que el anterior, a través de la fosa nasal lesionada.

- Pinzar los dos extremos juntos.

- Atar los dos extremos de una de las cintas a la punta del catéter que pasa por la fosa lesionada

- Tirar del catéter y de la cinta desde la nariz y dejar la segunda cinta colgando fuera de la boca, para utilizarla al retirar el taponamiento.

- Tirar suavemente del otro catéter para llevar hacia adelante el velo palatino y así facilitar el paso al taponamiento.

- Tirar del tapón hacia la parte posterior de la fosa nasal, empujando a través del paladar blando con el dedo.

Modo de hacer el taponamiento posterior

- Asegurar el taponamiento posterior. Anudar las cintas sobre un rollo dental, contra los orificios nasales.

- Tratar al paciente con anti congestivos y antibióticos por vía oral.

Retirada del taponamiento 


\section{Taponamiento nasal en epistaxis posterior}

Vol. 3, núm. 3., (2019)

María José Cajas Romero; Pedro Andrés Duque Torres; Margarita Alexandra Guagalango Cabrera; Karen Paola Barragan Arias

- El taponamiento posterior se deja durante 3-4 días; el anterior, 2 días.

- Quitar el taponamiento anterior.

- Cortar las cintas atadas al rollo dental.

- Extraer suavemente, a través de la boca, el tapón de la nariz, utilizando las cintas que se dejaron con este fin.

Si se reproduce la hemorragia.

- Volver a taponar.

- Consultar con el otorrinolaringólogo y considerar la posibilidad de ligadura de vasos (carótida externa, maxilar interna o etmoidal anterior). (Departamento de cirugia y radiologia y medicina fisisca, 2007)

El taponamiento nasal postero-anterior, puede ser realizado de 2 formas diferentes:

1) Se toman 2 o 3 torundas de gasa (dependiendo del tamaño de la coana del paciente) y se conforma un paquete que debe tener 4 cabos de hilos de seda bien gruesos, es decir, 2 hacia un extremo y 2 hacia el extremo opuesto. Previa anestesia tópica, se introduce una sonda por la fosa nasal sangrante hasta alcanzar la orofaringe, se practica una orofaringoscopia y se obtiene la sonda con una pinza de bayoneta, se fijan los hilos al extremo más distal de la sonda y se le comienza a retirar por la fosa nasal. El tapón de gasa es ayudado con el dedo índice del médico, hasta que este es impactado en la coana y se obtienen 2 hilos por la nariz y 2 quedan por boca, se traccionan los hilos que se obtuvieron por las fosas nasales (con la participación de un ayudante) y se procede a la colocar un taponamiento nasal 


\section{Taponamiento nasal en epistaxis posterior}

Vol. 3, núm. 3., (2019)

María José Cajas Romero; Pedro Andrés Duque Torres; Margarita Alexandra Guagalango Cabrera; Karen Paola Barragan Arias

anterior. Se sitúa una torunda frente a la narina y se anudan los 2 cabos de los hilos, mientras que los otros dos cabos que quedaron por la boca se fijan a la mejilla con esparadrapo, los que facilitarán la retirada del taponamiento nasal posteriormente.

2) Se utiliza una sonda vesical de Foley, que se introduce por la fosa nasal sangrante hasta llegar a la nasofaringe, insuflando el balón distal, se hace tracción de la sonda y se atasca dicho balón en la coana, después se rellena la fosa nasal con gasa (taponamiento anterior), se coloca una torunda de gasa delante de la narina (ala nasal) y se fija la sonda con hilo de seda. Este método no resulta difícil de realizar y se obtienen excelentes resultados.

Afortunadamente, la gran mayoría de los pacientes pueden resolver su desagradable cuadro de sangrado en el nivel primario de atención con los métodos enunciados, y aquellos que no lo logran, requieren de derivación no programada (remisión) al nivel secundario de atención para la aplicación de otros métodos más agresivos, es decir, cirugía (ligadura de vasos arteriales), que precisa de personal especializado. Otros recursos terapéuticos empleados en la epistaxis, sobre todo en el nivel secundario de atención, son: la embolización, la endoscopia con cauterización, la dermoseptoplastia, el catéter-balón, entre otros. (Moreno, Figueroa, \& Díaz, 2007)

Taponamiento anteroposterior con sonda de balón

Es un proceder sencillo, rápido y efectivo, requiere de poco material y tiene la ventaja de que puede realizarse en situaciones extremas como en guerras, catástrofes y otros desastres similares. 


\section{Taponamiento nasal en epistaxis posterior}

Vol. 3, núm. 3., (2019)

María José Cajas Romero; Pedro Andrés Duque Torres; Margarita Alexandra Guagalango Cabrera; Karen Paola Barragan Arias

Para ello se necesitan los siguientes elementos:

- Sonda de Foley con balón de 10 a 15 ml de capacidad.

- Jeringuilla tipo Luer para inflar el balón.

- Tiras de gasa vaselinadas o con pomada antibiótica y torundas del mismo material.

Taponamiento anteroposterior convencional (con gasa)

Este proceder terapéutico es considerado clásico dentro de la especialidad y para su realización tendremos que disponer de los siguientes materiales:

- Una sonda de Nélaton número 10 o 12

- Espéculo nasal

- Pinza de bayoneta

- Hebras de hilo de seda trenzada o hilo de poliéster calibre 1

- Tiras de gasas vaselinadas y torundas

- Depresor de lengua. (Moreno Rajadel, Figueroa Hernández, \& Díaz, 2007)

Complicaciones para el Taponamiento Nasal

- Rinitis aguda

- Sinusitis maxilar y frontal

- Hemotímpano

- Otitis media aguda

- Bacteriemia 
María José Cajas Romero; Pedro Andrés Duque Torres; Margarita Alexandra Guagalango Cabrera; Karen Paola Barragan Arias

- Dificultad Respiratoria

- Necrosis del tabique y de las alas nasales

- Intoxicación por lidocaína o tetracaina

- Intoxicación por cocaína

- Otalgia

- Cefalea

- Sequedad de boca y garganta

- Epifora

- Edema nasal

- Rinorrea serosanguinolenta

- Febrícula

- Dificultad para deglutir. (Freyre Serentill, 2011)

\section{Indicaciones post taponamiento posterior}

- Hospitalizar al paciente: reposo- signos vitales.

- Mantener vía venosa.

- Posición semi sentado.

- Régimen blando y papillas.

- Antibióticos siempre (Amoxicilina, es una alternativa)

- Sedación y analgésicos.

- Manejo de patología agregada (hipertensión arterial, alteraciones renales, hepática, etc.)

- Transfusión de sangre: Hematocrito menos de $20 \%$. 


\section{Taponamiento nasal en epistaxis posterior}

Vol. 3, núm. 3., (2019)

María José Cajas Romero; Pedro Andrés Duque Torres; Margarita Alexandra Guagalango Cabrera; Karen Paola Barragan Arias

También se puede optar por usar en taponamientos de subgalato de bismuto, que es un polvo que se usa como pasta adherido a los algodones o gasa del taponamiento, y tiene propiedades antisépticas y favorece la coagulación. (Hernández, Hernández, \& Bergeret, 2005)

En concordancia con lo descrito en la literatura, la epistaxis posterior fue más frecuente en pacientes adultos $(96,9 \%)$, no encontrándose diferencia significativa por género. La principal causa de predisposición fue el uso de aspirina, seguida por causa posquirúrgica y uso de terapia anticoagulante oral (TACO). En nuestro estudio, el manejo definitivo fue quirúrgico en el 57,5\% de los casos, siendo el abordaje de la arteria enfonopalatina (AEP) la cirugía más frecuente $(84,2 \%)$

Si bien este estudio muestra una eficacia similar entre el manejo médico y quirúrgico en el control de la epistaxis, han sido descritas en numerosos estudios las ventajas de esta última intervención.

El manejo endoscópico nasal permite localizar el sitio de sangrado, cuando esto es posible se realiza cauterización del sitio de sangrado que no requiere de un taponamiento posterior. Sin embargo, esta técnica tiene menor tasa de efectividad que la ligadura de AEP probablemente por identificación inadecuada del sitio de sangrado. En la ligadura, la eficacia depende del control de las ramas de la AEP, por lo que la indicación de ligadura de la AEP incluye la imposibilidad de taponamiento efectivo por deformidad anatómica, produciendo una falla en la terapia no quirúrgica, lo que se define como epistaxis recurrente.

Actualmente la indicación de cirugía endoscópica nasal se ha considerado como alternativa al taponamiento dado que, al comparar con técnicas tradicionales no quirúrgicas, la 


\section{Taponamiento nasal en epistaxis posterior}

Vol. 3, núm. 3., (2019)

María José Cajas Romero; Pedro Andrés Duque Torres; Margarita Alexandra Guagalango

Cabrera; Karen Paola Barragan Arias

ligadura de AEP ha demostrado mejor satisfacción del paciente, reducción de la estadía hospitalaria y los costos asociados. (Retuert et al., 2017)

\section{Conclusión.}

En lo que va del siglo XXI se ha experimentado una significativa expansión en alternativas para el manejo de las epistaxis. Si bien es cierto, las tradicionales técnicas de taponamiento nasal aún se realizan con éxito, se han incorporado al tratamiento nuevas tecnologías como el uso de materiales de tipo coloides para taponamiento, el uso de endoscopía, fibra óptica, láser y técnicas de embolización selectiva. En el presente estudio se describió la experiencia en el manejo endoscópico de pacientes con epistaxis posterior en lo transcurrido del presente siglo. (Alzérreca, León, Boettiger, \& Naser, 2012)

\section{Bibliografía.}

Alzérreca, E., León, K., Boettiger, P., \& Naser, A. (Agosto de 2012). Tratamiento endoscópico de las epistaxis posteriores. Experiencia del Hospital Clínico de la Universidad de Chile entre los años 2007 y 2011. Revista de otorrinolaringología y cirugía de cabeza y cuello, 72(2), 169-174. doi:10.4067/S0718-48162012000200011

Calderòn, D., Mairena, A., \& Mata, C. (2014). Epistaxis: Generalidades y Manejo en atencion Primaria de Salud. Revista medica costa rica y centroamerica, 71(610), 219-223.

Departamento de cirugia y radiologia y medicina fisisca. (2007). Epistaxis: diagnóstico y alternativas terapéuticas actuales. Rev. Hosp. Clín. Univ. Chile, 18(1), 227 - 38.

Freyre Serentill, J. (2011). EPISTAXIS. Cuba, La Habana: ORL.

Hernández, M., Hernández, C., \& Bergeret, J. (2005). Epistaxis.Consideraciones generales y manejo clínico. Cuadernos de cirugia, 19, 54-59. Recuperado el 15 de 09 de 2019, de http://mingaonline.uach.cl/pdf/cuadcir/v19n1/art09.pdf

Moreno Rajadel, R., Figueroa Hernández, A., \& Díaz, A. (2007). Epistaxis. Consideraciones sobre el tratamiento clínicoy terapéutico en la atención primaria de salud. Rev Cubana Med Gen Integr, 23(4), 1-10. Recuperado el 15 de 09 de 2019 


\section{Taponamiento nasal en epistaxis posterior}

Vol. 3, núm. 3., (2019)

María José Cajas Romero; Pedro Andrés Duque Torres; Margarita Alexandra Guagalango Cabrera; Karen Paola Barragan Arias

Moreno, R., Figueroa, A., \& Díaz, A. (2007). Epistaxis. Consideraciones sobre el tratamiento clínico. Revista cubana de medicina general integral, 23(4), 1-10. Recuperado el 15 de 09 de 2019, de http://scielo.sld.cu/pdf/mgi/v23n4/mgi07407.pdf

Naser, A., \& Aedo, C. (2007). Epistaxis: diagnóstico y alternativas terapéuticas actuales ${ }^{\circ}$. Revista Hospital Clínico Universidad de Chile, 18(38), 227- 238. Recuperado el 15 de 09 de 2019, de https://www.redclinica.cl/Portals/0/Users/014/14/14/epistaxis.pdf

Sociedad epañola de medicina interna. (10 de Septiembre de 2019). SEMI. Recuperado el 15 de 09 de 2019, de SEMI: https://www.fesemi.org/informacion-pacientes/conozca-mejor-suenfermedad/epistaxis 